\title{
Dynamic rayed aurora and enhanced ion-acoustic radar echoes
}

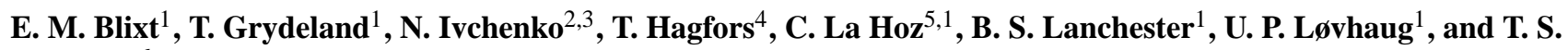 \\ Trondsen ${ }^{6}$ \\ ${ }^{1}$ Dept. of Physics, University of Tromsø, N-9037 Troms $\varnothing$, Norway \\ ${ }^{2}$ School of Physics and Astronomy, University of Southampton, UK \\ ${ }^{3}$ Alfvén Laboratory, KTH, Stockholm, Sweden \\ ${ }^{4}$ Max-Planck-Institut für Aeronomie, Max-Planck-Str. 2, 37191 Katlenburg-Lindau, Germany \\ ${ }^{5}$ Cornell University, Earth and Atmospheric Science Department, Ithaca, USA \\ ${ }^{6}$ Institute for Space Research, Calgary, Alberta, Canada
}

Received: 13 October 2003 - Revised: 9 February 2004 - Accepted: 14 May 2004 - Published: 31 January 2005

Part of Special Issue "Eleventh International EISCAT Workshop"

\begin{abstract}
The generation mechanism for naturally enhanced ion-acoustic echoes is still debated. One important issue is how these enhancements are related to auroral activity. All events of enhanced ion-acoustic echoes observed simultaneously with the EISCAT Svalbard Radar (ESR) and with high-resolution narrow field-of-view auroral imagers have been collected and studied. Characteristic of all the events is the appearance of very dynamic rayed aurora, and some of the intrinsic features of these auroral displays are identified. Several of these identified features are directly related to the presence of low energy $(10-100 \mathrm{eV})$ precipitating electrons in addition to the higher energy population producing most of the associated light. The low energy contribution is vital for the formation of the enhanced ion-acoustic echoes. We argue that this type of aurora is sufficient for the generation of naturally enhanced ion-acoustic echoes. In one event two imagers were used to observe the auroral rays simultaneously, one from the radar site and one $7 \mathrm{~km}$ away. The data from these imagers shows that the auroral rays and the strong backscattering filaments (where the enhanced echoes are produced) are located on the same field line, which is in contrast to earlier statements in the litterature that they should be separated.
\end{abstract}

Key words. Ionosphere (Auroral ionosphere; Plasma waves and instabilities; Particle acceleration)

\section{Introduction}

So-called naturally enhanced ion-acoustic echoes stand out as a significant problem in the understanding of incoherent scatter (IS) radar data. They are easily distinguishable from normal thermal back scattering because of the very strong enhancement of one, or both, ion-acoustic shoulders of the returned spectrum (corresponding to an ion-acoustic wave traveling away from, or towards the radar, or both), and their very dynamic appearance. They are different from the strong coherent back scattering from solid objects, such as satellites, as they are smeared out in the field-aligned direction.

The first reported observations of such enhanced ionacoustic echoes stem from the Haystack observatory (Foster et al., 1988). Similar observations were later made by the EISCAT facilities (Collis et al., 1991; Rietveld et al., 1991). Based on observations, three main mechanisms evolved to explain the observations (Sedgemore-Schulthess and St.-Maurice, 2001). Two of them are based on streaming instabilities, and rely on strong relative drift between two thermal populations (ion-ion and ion-electron). A third explanation is based on the decay of Langmuir waves (due to a bump-in-tail instability) into a secondary pair of Langmuir and ion-acoustic waves. Very recently, the extremely small temporal and spatial scales of the enhancements were unraveled by Grydeland et al. $(2003,2004)$ by using the two antennas of the EISCAT Svalbard Radar (ESR) as an interferometer. Their results showed that a time resolution of $0.2 \mathrm{~s}$ is required to resolve the enhanced radar echoes, and that this scattering can arise in filaments narrower than a few hundred meters in the direction perpendicular to $B$, even at several hundred kilometers altitude. Evidence that the scattering cross section is increased at least 4-5 orders of magnitude, and that up- and down-shifted echoes can be enhanced simultaneously, are strong support for the Langmuir wave decay model (Grydeland et al., 2003, 2004). We explicitly take this model as the working hypothesis for explaining the observed enhanced echoes. 
Table 1. List of events.

\begin{tabular}{lllll}
\hline & Date & $\begin{array}{l}\text { Time } \\
\text { (UT) }\end{array}$ & $\begin{array}{l}\text { Time res. (s) } \\
\text { Radar / imager }\end{array}$ & Notes \\
\hline 1 & $24-01-1998$ & $07: 00$ & $10 / 0.033$ & Sedgemore-Schultess \\
2 & $24-01-1998$ & $07: 06$ & $10 / 0.033$ & et al. (1999) \\
3 & $17-01-2002$ & $06: 46$ & $\leq 0.2 / 0.033$ & Grydeland et al. (2003, 2004) \\
4 & $26-01-2003$ & $06: 53$ & $\leq 0.2 / 0.04$ & \\
$X$ & $30-01-2003$ & $07: 10$ & $\leq 0.2 / 0.04$ & No enhanced echoes \\
\hline
\end{tabular}

Earlier observations suggest that enhanced ion-acoustic echoes are associated with intense red aurora, and are restricted to the boundaries of (stable) auroral arcs (Collis et al., 1991; Rietveld et al., 1991; Cabrit et al., 1996), while newer observations indicate that the enhancements accompany rayed aurora and coronal structures. SedgemoreSchulthess et al. (1999) were the first to utilize a highresolution narrow field-of-view imager in their coordinated observations of enhanced echoes at ESR. They noticed that the radar beam can, within a single radar integration period ( $10 \mathrm{~s}$ in that case), be located both outside and inside aurora. They suggest that coronal forms are both necessary and sufficient for the formation of enhanced up-shifted shoulders, and that luminosity within the radar beam is necessary, but not sufficient, to generate enhanced down-shifted shoulders. Grydeland et al. (2004) were the first to resolve fully both the radar enhancements and the aurora. The spatial and temporal resolution of the earlier observations (before 1999) are very limited, considering the newer findings that the enhancements are varying on a subsecond time scale, and that the aurora is very dynamic. Such active aurora cannot be studied properly using photometer measurements of $630 \mathrm{~nm}$ emissions (Collis et al., 1991), all-sky imagers (Rietveld et al., 1991), or EISCAT estimates of auroral position (Cabrit et al., 1996).

\section{Instrumentation}

\subsection{Radar}

The EISCAT Svalbard Radar (ESR), situated at $78^{\circ} 9^{\prime} \mathrm{N}$, $16^{\circ} 2^{\prime} \mathrm{E}$, is described by Wannberg et al. (1997). Four events have been analysed from dates listed in Table 1. Different radar programs were used in each, all looking in the fieldaligned direction. During events 1 and 2, a long pulse program, gup0, was used. Standard EISCAT signal handling was in use, which dumps raw data in pre-integrated 10-s packages. For event 3 , the gup 0 program was used, with an additional receiving system, the MIDAS-W receiver (Holt et al., 2000), in operation. This samples and stores the signal directly (without any pre-integration) on a large hard disc. This technique allows the received signal to be analysed at very high temporal resolution (in theory limited by the pulse length of the transmitted pulse). For event 4, a specially de- signed long pulse program, $l t 1 h$, was used. This is a combined ion-line/plasma-line program, designed specifically for a MIDAS-W type receiver for the ion-lines. This program has a very large range coverage, from about 300 to $1600 \mathrm{~km}$, which was chosen to investigate the height regime of enhanced ion-acoustic echoes.

\subsection{Imager}

The different events used different imaging facilities. The common features of these are that they all record in standard video (digital and analog) format, they use imageintensifiers, fiber-optically coupled to the CCDs (Charge Coupled Device) to achieve the highest possible sensitivity, and the camera units are equipped with standard C-mount lens adaptors so that a wide range of lenses can be used. The response of the imagers varies with wavelength, so that absolute brightness of auroral emissions cannot be determined. In most of the events, cutoff filters have been used to eliminate long-lived emissions.

Events 1-3 were covered by the University of Calgary Portable Auroral Imager (PAI) located at the Auroral Station in Adventdalen, about $7 \mathrm{~km}$ north of ESR, at $78^{\circ} 12^{\prime} \mathrm{N}$, $15^{\circ} 50^{\prime} \mathrm{E}$. The camera was pointed towards the magnetic zenith. For events 1 and 2 a lens with a field-of-view of $15^{\circ} \times 12^{\circ}$ was used. A $675 \mathrm{~nm}$-cutoff filter was mounted to suppress the slow $630 \mathrm{~nm}$-(red) emission and focus on the prompt $\mathrm{N}_{2}$ and $\mathrm{N}_{2}^{+}$emissions. For event 3, a shorter lens with a viewing angle of about $31^{\circ}$ by $23^{\circ}$, without cutoff filter, was used.

During event 4 (and $X$ ), two imagers were used. The Spectrographic Imaging Facility (SIF) is a platform of instruments at the Auroral Station run by University of Southampton and University College, London, and includes the narrow angle imager $12^{\circ} \times 16^{\circ}$ used here (Lanchester et al. (2003)). The Optical Digital Imager (Odin) of University of Troms $\varnothing$ is a new imager mounted at the ESR site. As these are the first reported observations from this imager, more detail is given here. The core of the imager is a video camera (XYBION ISS-750), where a Generation 3 extended blue image intensifier (64 line-pairs $/ \mathrm{mm}$ ) is used. The CCD has $756 \times 581$ pixels on $8.32 \times 6.29 \mathrm{~mm}^{2}$, and a full well capacity of about 100 kilo-electrons per pixel. The camera outputs a standard PAL video signal, 25 frames/s, with a resolution of 525 horizontal lines. The video signal is digitized in real time and converted 




Fig. 1. Typical images from three of the events, and a fourth image, in the lower right frame, which shows a coronal aurora from event $X$ with no associated enhanced ion-acoustic echoes. The upper left frame is from event 1 , the upper right from event 3 , and the lower left frame from event 4.

to the (PAL) DV standard and stored on DVCAM tapes using a SONY DSR-1500P digital video recorder. A F0.95 50-mm lens was mounted, yielding a $14.3^{\circ} \times 10.9^{\circ}$ field of view. On both imagers a 650-nm cutoff filter was used.

\section{Observations}

Only four of all events known to the authors (out of a total of more than $300 \mathrm{~h}$ of observations) are found to have observations of enhanced ion-acoustic echoes when the sky was clear and high-resolution narrow field-of-view auroral imagers were operating. These are presented in Table 1. These auroral sightings are compared with an additional event (event $X$ in Table 1) where coronal aurora was observed, but no enhanced ion-acoustic echoes. They all occur in the pre-noon (MLT) sector. Selected images from three of the events, plus one from the additional event, are shown in Fig. 1.

The geophysical background is similar for all events, with moderate activity ( $K_{p}$ is $3,3,3$, and 4 , respectively), and all observations are made in the proximity of the cusp. In all events, the meridian scanning photometer (MSP) show strong red emissions moving north, associated with softparticle precipitation. In events 1 and 2, the radar is situated slightly south of this dominant red region, while for events 3 and 4 it is within it. The poleward moving red aurora is clearly seen in the top panel of Fig. 2.

\subsection{Radar observations}

In events 1 and 2 , there are significant changes between each 10 -s data dump, indicating that the physical mechanism is acting on a time scale much shorter than $10 \mathrm{~s}$, but it is active for 20 to $30 \mathrm{~s}$.

The activity in the enhanced ion-acoustic echoes for event 3 is shown in Fig. 1 in Grydeland et al. (2003), and is not repeated here. As in events 1 and 2, this lasts for about $20 \mathrm{~s}$, but the high time resolution allows the dynamics to be fully resolved (Grydeland et al., 2004). 


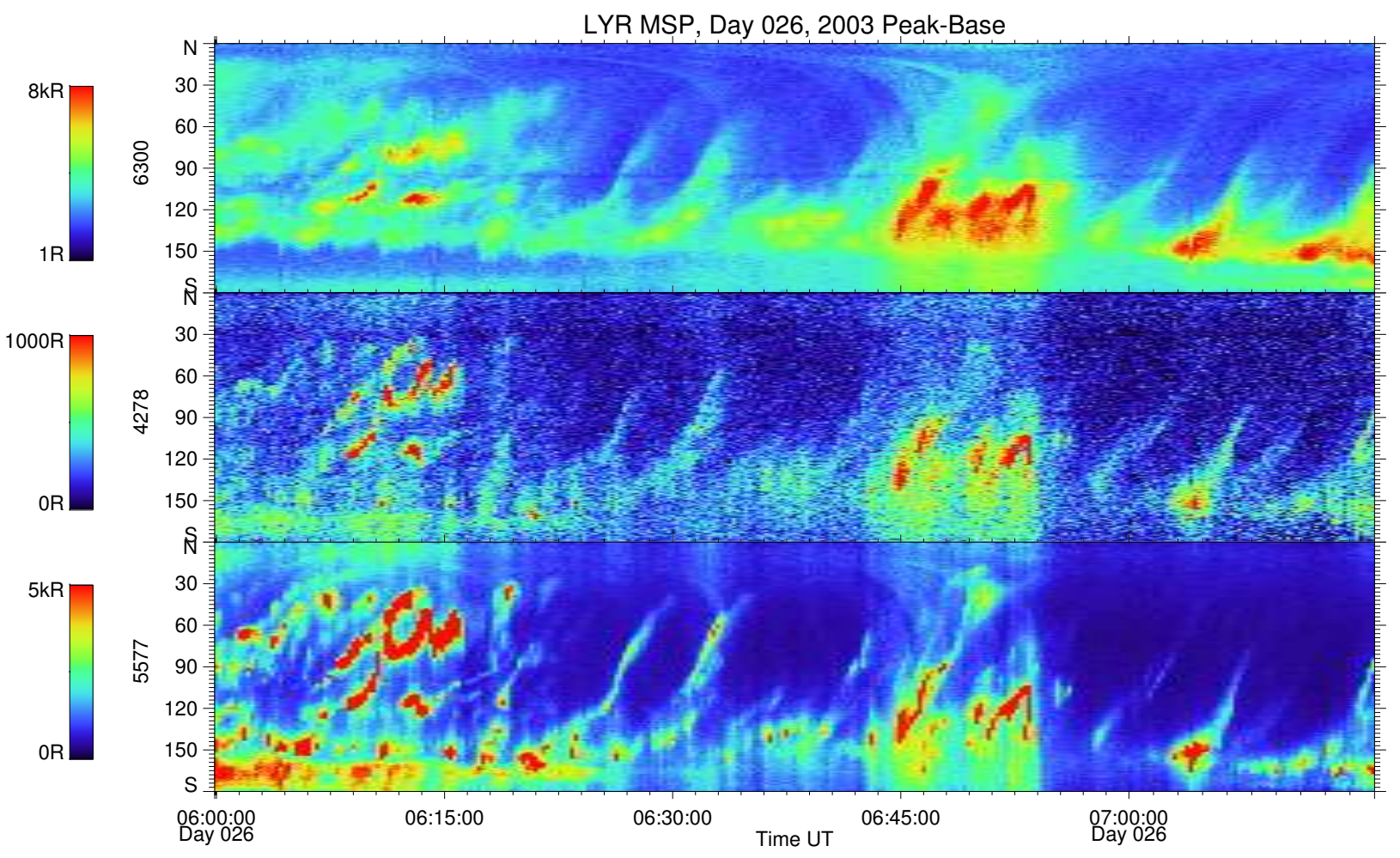

Fig. 2. Meridian scanning photometer data from event 4 (26 January 2003, 06:53 UT), The panels show the 630.0-nm (red), 427.8-nm (blue) and 557.7-nm (green) emissions in $\mathrm{kR}$, from top to bottom, respectively. Around the time of the event there are fairly strong red emissions, which in the absence of other optical instruments, easily could have been interpreted as northward moving red arcs. The radar is pointing just south of the $90^{\circ}$ scanning elevation angle.

During event 4 , the radar program $(l t 1 h)$ covered a slightly different range regime, observing also at much greater altitudes than earlier, at the expense of lower range resolution. No ion-acoustic shoulders were observed to be enhanced above $1400 \mathrm{~km}$ in that event, and very few even at $1200 \mathrm{~km}$. The middle spectra in Fig. 4 shows one of the highestreaching enhanced shoulders during that event. The upper panel of Fig. 3 shows the evolution of the power (per unit frequency), for two different range regions and for up-/downshifted ion-acoustic shoulders, in time (arbitrary units). The event covers approximately $40 \mathrm{~s}$, and similar to event 3 , the activity of the enhanced shoulders is more pronounced and powerful in the upper height regime (above $550 \mathrm{~km}$ ), while the background level is much higher at lower altitudes. As in event 3 , enhancements observed below $550 \mathrm{~km}$ are always simultaneous with (small) enhancements above 550, but quite often enhancements are observed above $550 \mathrm{~km}$ and not below it. In event 3 and 4 the down-shifted shoulder is also generally more strongly enhanced than the up-shifted in the higher range regime, but the two shoulders are similarly enhanced at lower ranges. However, the enhancements are less intensive in event 4 than in event 3.

Two typical enhanced echoes from events 3 and 4 are shown in Fig. 4, together with the normal thermal spectrum observed during event $X$.

\subsection{Imager observations}

The rapidly moving rays in the four events are very different from arcs in which rays form part of a curtain and are more stable (Jussila et al., 2003). Crude estimates yield horizontal velocities of several $\mathrm{km} / \mathrm{s}$ when mapped to an altitude of $105 \mathrm{~km}$. Another significant feature we observe is the occurrence of patches of enhanced luminosity in the rays that almost move independent of the rays. The enhanced luminosity moves from ray to ray, and also along the rays. In these very luminous parts the imagers are easily saturated, thus losing contrast and the possibility of identifying individual rays. Sometime these luminous parts move up and down in a semi-periodic way as if the rays are "pumping" (approximately around $5 \mathrm{~Hz}$, which corresponds roughly to flickering aurora).

A third important signature is how very clear the rays and structures appear in the images, which makes it likely that they stem from prompt emissions. This is further emphasized by the fact that event 3 was observed in white light, while cutoff filters that remove the slow 630-nm emissions were used in the others. Still the rays appear very distinct. Because the sensitivity of the imagers is decreasing rapidly in the near-infrared part of the spectrum, the emissions seen are most likely from the prompt $\mathrm{N}_{2}$ (first positive) and $\mathrm{N}_{2}^{+}$ (Meinel) emissions. 

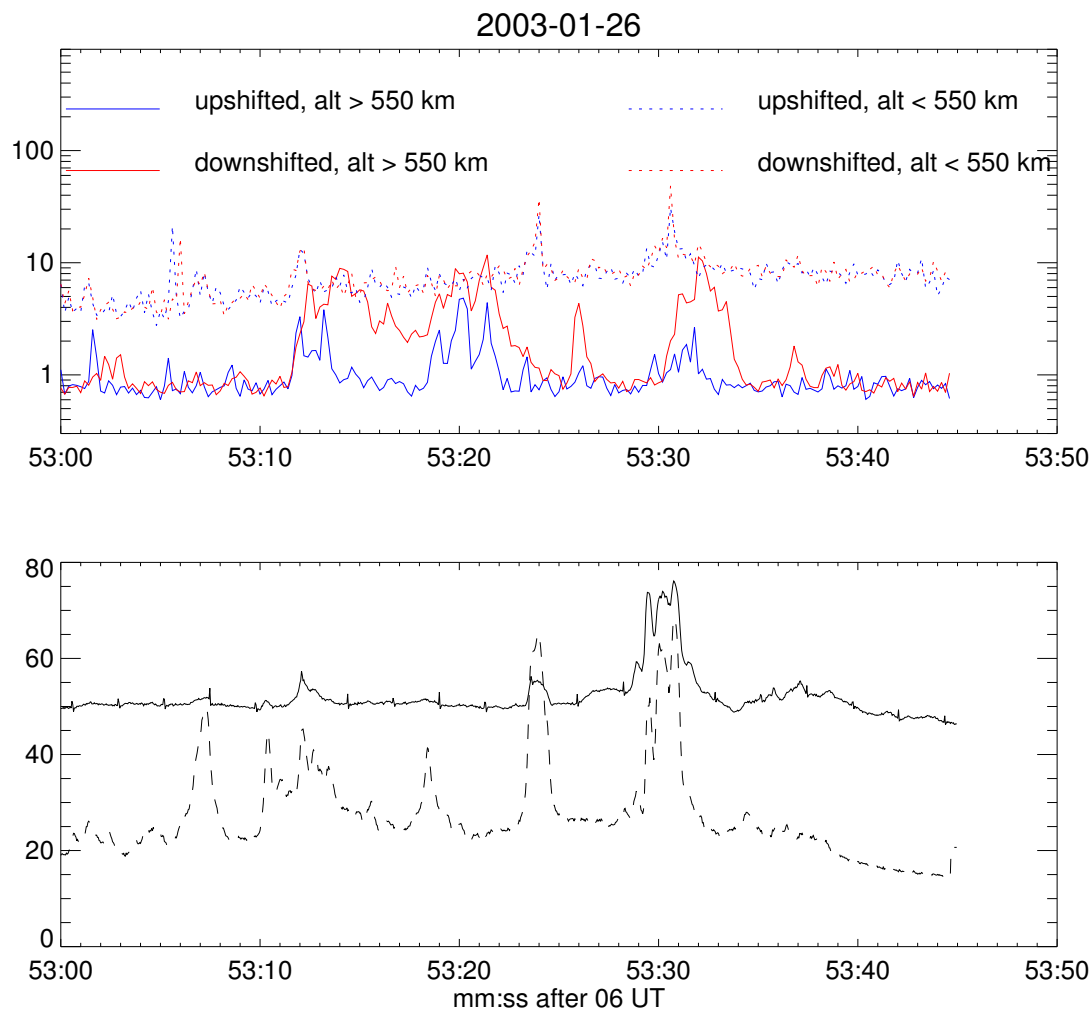

Fig. 3. Time series of radar (upper panel) and imager (lower panel) data from event 4. The radar data is power per unit frequency at the peak of the ion-acoustic shoulder, for two different height regions and for the up- and down-shifted shoulder, as a function of time (arbitrary units). In the lower panel the total "in-beam" luminosity, measured by the imager (arbitrary units), is shown as a function of time. This event was covered by two imagers, and the dashed line in the lower panel shows the in-beam luminosity moves from the SIF imager mounted at the Auroral Station, while the solid line is from the Odin imager mounted at the ESR site. The view from the ESR was slightly obscured by thin cloud, and the periodic spikes in the solid line are due to reflections from this layer of a warning lamp.

A significant feature in event 3 is the appearance of socalled flaming aurora (Scourfield and Parsons, 1969; Cresswell, 1969; Omholt, 1971). This is a $p_{2}$ type pulsating aurora where surges of luminosity move up along the magnetic field, and thus converge in the magnetic zenith. They are usually attributed to significant energy dispersion in the precipitating electron population, where the higher energy electrons reach the ionosphere first, and penetrates deeper, while the lower energy particles are stopped at higher altitude. If the acceleration process is limited in time, then as the luminosity disappears at low altitude, it remains for a brief time at higher altitude, and the whole ray seems to be drawn out from the ionosphere. The flaming appearance can be considered a special case of the "pumping" effect seen in all the events, but where the dispersion is more pronounced. The scale of the structures and the signatures of a mixed energy content in the precipitating electrons all indicate that the aurora is not due to a large inverted- $\mathrm{V}$ event. This conclusion, and why it may be important for the generation of enhanced radar echoes is further discussed in Sect. 4.

The position of the radar beam in the imager field-of-view can be determined for a given height (here $105 \mathrm{~km}$ is used). The total luminosity within this circular region of each image (in-beam luminosity) can be used in comparative studies of radar and optical signatures. In event 4 two imagers were used. The in-beam luminosities from these are shown in the lower panel of Fig. 3, where the dashed line is from the SIF imager at the Auroral Station and the solid line is from the Odin imager at the ESR site. The ESR site was slightly obscured by thin cloud, but the aurora could be seen through it. The periodic spike in the luminosity from the Odin imager is caused by a warning light at the entrance to the ESR site. The in-beam luminosity from the SIF imager (dashed line) shows much more activity than the Odin imager, which could be partially due to the thin cloud cover above the ESR site which filters out weak auroral emissions. Another difference is that the dashed line follows more closely the activity of the power in the up-shifted shoulder at altitudes above $550 \mathrm{~km}$, while the solid line is more similar to the evolution in power of both shoulders at altitudes below that.

The auroral display from event $X$ is just as active as the others, but the structures in the aurora are more diffuse, and no distinct ray structures moving rapidly could be identified, nor any pulsations or flaming aurora.

Based on the above observations we use the following 3 signatures to characterize the aurora present during enhanced ion-acoustic echoes: 

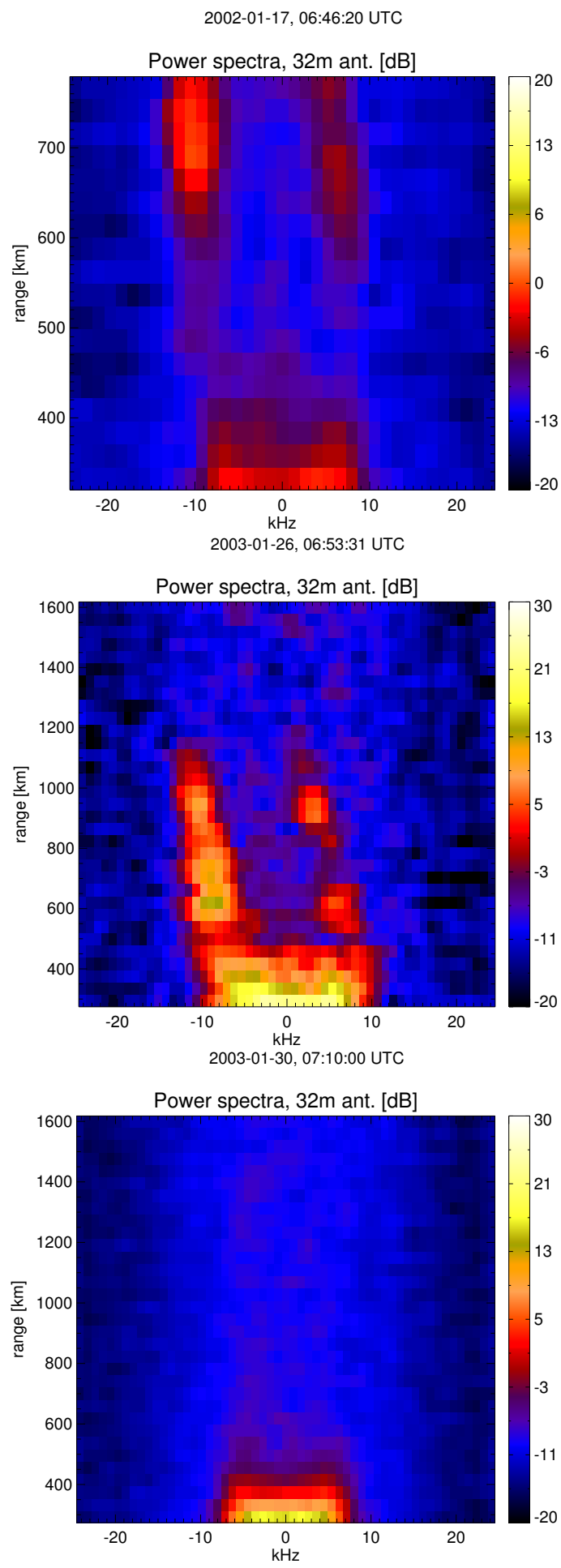

Fig. 4. Three spectra received at the ESR. At the top is an enhanced spectra from event 3 , and in the middle is an enhanced spectra from event 4 . The third spectra at the bottom is a normal thermal spectrum from event $X$, when no enhancements were observed at all. Images from the auroral imagers taken during the same times are shown in Fig. 1. The data has not been background subtracted or corrected for attenuation with range squared.
1. Very dynamic rayed aurora, with apparent horizontal motion of rays at $105 \mathrm{~km}$ altitude of several $\mathrm{km} / \mathrm{s}$.

2. Individual rays appear very distinct, even in observations in white light where one might expect contamination from long-lived emissions.

3. Superimposed on the ray structure are patches of enhanced luminosity, which move both horizontally, crossing from ray to ray, and vertically, along the rays. This introduces a secondary visual appearance of "pumping" and "flaming".

Further studies on these dynamic rays must be performed, and more quantitative signatures must be isolated to aid the identification of events similar to those reported here.

\section{Discussion}

The following discussion consists of two topics: 1) that enhanced ion-acoustic echoes are associated with dynamic rayed aurora, and 2) that the scattering region (causing the enhanced radar spectrum) is on the same field line as the auroral rays, or very close.

Red auroral emissions are seen in the MSP data during all the events. This is also a characteristic of earlier observations of enhanced ion-acoustic echoes from EISCAT Troms $\varnothing$. For example, Collis et al. (1991) report the passage of a strong rayed red aurora during their observations, and locate the enhanced echoes at the borders of this aurora. Whether such reports have enough time resolution to determine the full dynamics of the event, so that the aurora and the enhanced echoes really can be separated, is not known. Without the high-resolution imagers used in this report, the auroral activity could simply have been interpreted as poleward moving red auroral forms. All the earlier observations were made on the mainland, while all the events presented here are from the 06:00-07:00 UT interval on Svalbard (high magnetic latitude). Therefore, the two sets of observations are very different in context. Rietveld et al. (1996) showed there was a minimum in the occurrence rate in the morning hours (UT) for the mainland observations of enhanced echoes, and a maximum in the afternoon/evening, and that the echoes were preferably found during geomagnetically disturbed times. However, recent statistical studies by Ivchenko and Marklund (2001, 2002) and Neubert and Christiansen (2003) shows that small-scale activity (Alfvén wave activity and small-scale field-aligned currents) are preferably found in the cusp/cleft region, and during periods of high geomagnetic activity, also in the premidnight sector at lower magnetic latitude. Thus, although the setting is very different between mainland observations in the premidnight sector and Svalbard observations in the prenoon sector, the geophysical setting, in the form of small-scale activity, can be very similar. 


\subsection{Auroral rays and enhanced radar echoes}

Sedgemore-Schulthess et al. (1999) claimed that coronal forms were necessary and sufficient for the formation of enhanced up-shifted shoulders. Event $X$, where coronal forms are present, but there are no enhanced echoes, shows that that is not the case.

For the formation of enhanced ion-acoustic echoes from the Langmuir wave decay model (Forme, 1993, 1999), a "seed" high energy population (high compared to the thermal plasma) is needed (to initiate the Langmuir turbulence from a bump-in-tail instability). For the ESR radar, at $500 \mathrm{MHz}$, the initial seed population must have an energy of $10-100 \mathrm{eV}$ (Forme, 1993). This energy level is very low when compared to the typical energy of precipitating electrons (several $\mathrm{keV}$ ). Thus, it is important that the precipitating population have a low energy component. The very nature of the observed aurora indicate that there are precipitating sub-keV electrons. Ray structures in aurora are closely associated with electron density profiles produced by a precipitating population with Maxwellian energy spectrum, rather than that of a monoenergetic beam (Ivchenko et al., 2004).

Alfvén wave acceleration of electrons is generally believed to cause the small-scale structures observed in aurora (Stasiewicz et al., 2000), and the observed aurora carries many signs of an Alfvénic acceleration (i.e. small scales and dynamic). Furthermore, dispersive Alfvén waves give rise to so-called suprathermal electron bursts, where the precipitating electron energy may extend up to $1 \mathrm{keV}$ (Andersson et al., 2002). Flaming aurora is another indicator that there is a mixture between high and low energy precipitating electrons.

Thus, we argue that the acceleration mechanisms for dynamic rayed aurora is of an Alfvénic type, which generates a significant number of lower energy electrons, in addition to the main bulk of high energy precipitating electrons. This low energy component (in this case, $10-100 \mathrm{eV}$ ) is vital for the formation of Langmuir turbulence in the upper ionosphere. Hence, the active rayed aurora is sufficient for enhanced echoes, but not necessary. Other mechanisms might yield the necessary $(10-100 \mathrm{eV})$ electron beams that could generate Langmuir turbulence.

\subsection{Localisation of scattering region vs. auroral rays}

From earlier observations on the mainland, we know that the occurrence rate of enhanced echoes increases with height above $250 \mathrm{~km}$, and maximizes at $500 \mathrm{~km}$ (Rietveld et al., 1996). Our observations stretch further out, and do not fit with the above observations. Instead, the observations in Fig. 3 show more enhanced echoes at altitudes above $500 \mathrm{~km}$.

Grydeland et al. (2003) showed that the coherent structures causing the enhanced echoes are extremely thin and filamented. As shown in Fig. 5, a structure within the radar beam at $800 \mathrm{~km}(\mathrm{~B})$ may be situated 7 to $10 \mathrm{~km}$ outside the beam at $100 \mathrm{~km}$ altitude, because of the angular width of

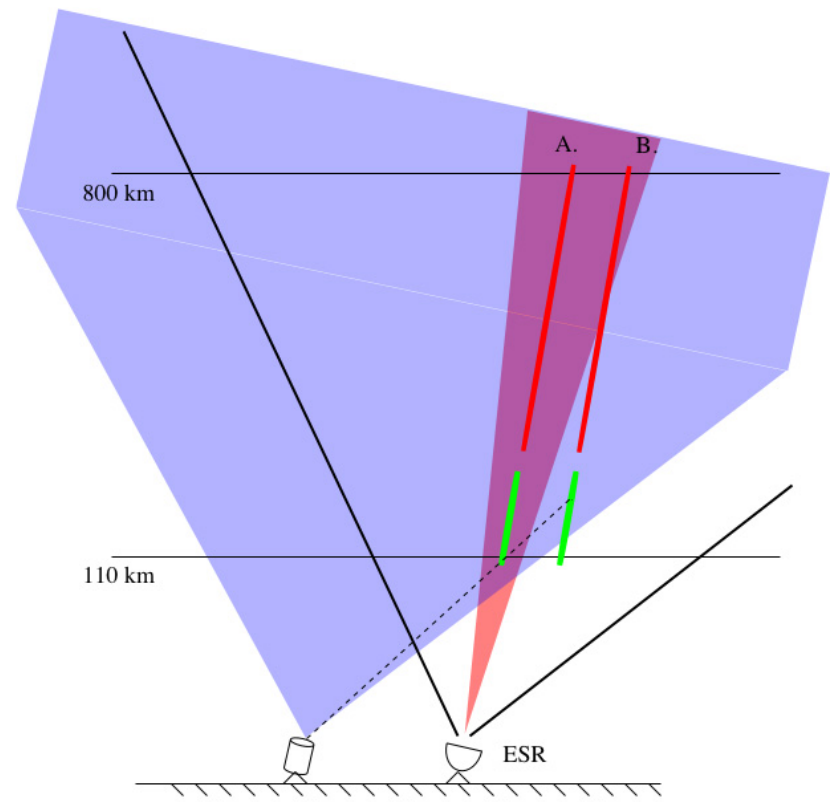

Fig. 5. A drawing of the imager/radar set-up used in the observations. The shaded red triangular region is the radar beam, while the larger shaded blue region is the field-of-view of the camera at the Auroral Station. The strong lines drawn from ESR shows the fieldof-view of the camera mounted there. Two different situations, A and $\mathrm{B}$, are shown. In A, both the enhanced radar echo (red) and the auroral ray (green) are within the radar beam at all altitudes, while they are partially outside in B. In the latter case, the aurora will appear to be "in-beam" at $105 \mathrm{~km}$ when viewed from the Auroral Station, as shown by the dashed line drawn from the imager through the radar beam.

$\sim 1.3^{\circ}$ of the radar beam. This can in part explain why the occurrence rate increases with range.

When viewing along the radar beam, from the Odin imager mounted at the ESR site, the in-beam luminosity would correlate with enhanced echoes observed both at low and high altitudes, if we assume that auroral rays and enhanced echoes occur on the same (or two very close) field line(s). This is the situation outlined in Fig. 5, by the red (the strong backscattering region) and green (auroral ray) structures under A. However, a structure only observed at high altitudes is not expected to correspond to any in-beam luminosity, as seen by the structures under B.

Furthermore, when the imager and radar are separated by several kilometers, there is an ambiguity in interpreting the optical signatures. The dotted line in Fig. 5 shows that there might be contributions to the measured light intensity from structures behind, but at higher altitudes (as well as from in front at lower altitudes). We can use this to our advantage; in situation B (in Fig. 5) there will be a correlation between high altitude enhancements and in-beam luminosity when seen from the off-radar imager, but not for the imager colocated with the radar.

In order to study this effect in the time series shown in Fig. 3, we have calculated the cross-correlation coefficient of 


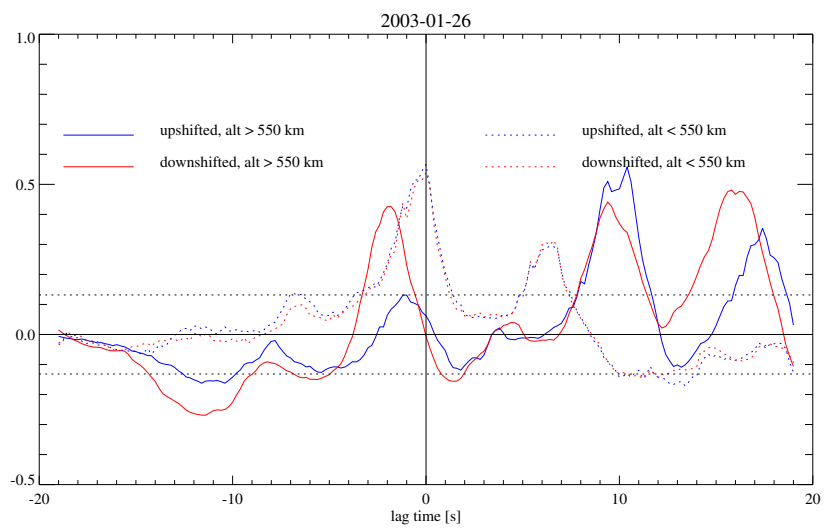

Fig. 6. Correlation between the in-beam luminosity and time series of received radar power from different regions of the radar spectrum. The in-beam luminosity here is taken from the Odin imager mounted at the ESR site.

the in-beam luminosity of the Odin imager (at ESR) and the power received by the radar at the peak of the ion-acoustic shoulder. Figure 6 shows the result. This is compared with the same analysis using the SIF imager data, shown in Fig. 7. Because the time series are only $45 \mathrm{~s}$ long, peaks in the crosscorrelation coefficient at time lags greater than $\pm 10 \mathrm{~s}$ have very low statistical significance.

As can be seen directly, the view from the Odin imager, Fig. 6 correlates better with the lower altitude radar power (dashed lines) than does the SIF imager (Fig. 7). In contrast, the SIF imager in-beam luminosity has a much better correlation with the high altitude received radar power, than does the Odin imager, especially for the down-shifted line (compare the red solid lines in Figs. 6 and 7). Thus, the activity of the auroral rays and enhanced scattering is aligned along a field line. A similar analysis, as shown in Fig. 6, was performed, using instead the luminosity within a ring surrounding the radar beam (with an outer radius twice that of the radar beam). If there was an offset between the scattering filament and the auroral ray, then the resulting correlation would be stronger than the result in Fig. 6. However, the resulting cross-correlation coefficients of such an analysis, although similar to that in Fig. 6, are about 5\% lower. This further supports the conclusion that the strong backscattering filaments are on the same field lines as the auroral rays, although at a much higher altitude (they can be separated by as much as $800-1000 \mathrm{~km}$ in altitude).

\section{Conclusions}

All known observations of enhanced ion-acoustic echoes which were coordinated with high-resolution narrow fieldof-view optical imagers have been reviewed. The four radar enhancement events last from 20 to $40 \mathrm{~s}$, but with large variations in the degree of enhancement within that interval. The auroral display during all the events shows a striking similarity, with pulsating and flaming auroral rays moving rapidly

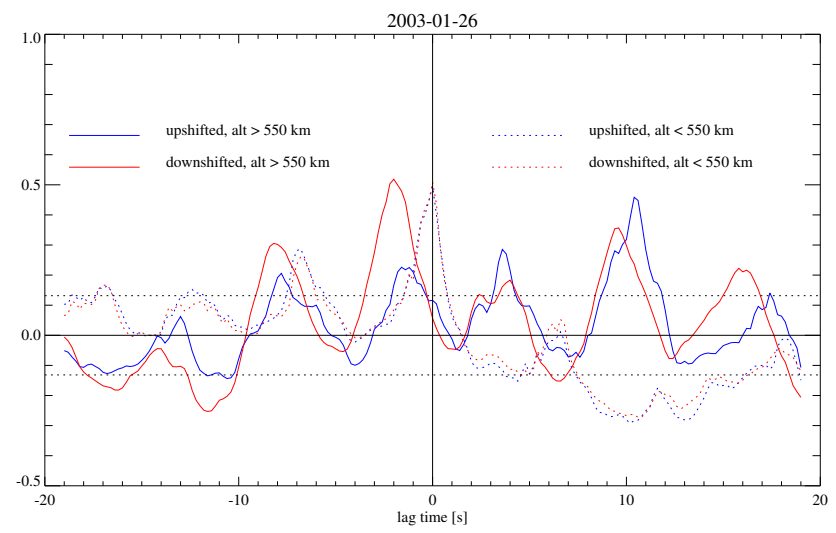

Fig. 7. Same type of correlation as in Fig. 6, from the same time, but the in-beam luminosity is taken from the SIF imager mounted at the Auroral Station.

across the field-of-view, in addition to strong red emissions in the background.

Three signatures of the dynamic rayed auroral displays were used to identify similar events.

1. Rays which move fast, up to several $\mathrm{km} \mathrm{s}$ at $105 \mathrm{~km}$ altitude, in the horizontal direction.

2. Individual rays that are distinct, even when observed in white light.

3. Patches of enhanced luminosity which move independently of the rays, giving rise to the visual appearance of "pumping" and "flaming".

The strong correspondence between enhanced ion-acoustic echoes and dynamic rayed aurora suggests that they are intimately linked. The small-scale and fast movements in the auroral display indicates that Alfvénic acceleration mechanisms are important. This, together with the extended altitude coverage of auroral rays and the red background emissions, shows that there is a significant part of low energy (sub $\mathrm{keV}$ ) electrons among the precipitating electrons. This low energy part is proposed to be a key component to the generation of enhanced ion-acoustic echoes via a nonlinear wavedecay of Langmuir waves (Forme, 1993).

Earlier observations have shown that enhanced ionacoustic echoes are related to (stable) red arcs, preferentially located on the edge of the arc. Such characteristics are inconsistent with the four reviewed events, because of their very dynamic structure. Simultaneous observations of auroral rays from two optical imagers separated by $7 \mathrm{~km}$, shows that the strong backscattering filaments are located on the same field line as the auroral rays, and not on the edge of an auroral arc.

Acknowledgements. The authors like to thank Dr. Anastasia "Molly" Stockton-Chalk and Jeffery M. Holmes for the MSP data analysis.

The EISCAT Scientific Association is supported by Centre National de la Recherche Scientifique of France, Max-Planck-Gesellschaft of 
Germany, Particle Physics and Astronomy Research Council of the United Kingdom, Norges Forskningsråd of Norway, Naturvetenskapliga Forskningsrådet of Sweden, Suomen Akatemia of Finland and the National Institute of Polar Research of Japan. This project has been supported through grant 147769/431 from the NFR of Norway.

Topical Editor M. Lester thanks P. Janhunen and V. Howells for their help in evaluating this paper.

\section{References}

Andersson, L., Ivchenko, N., Clemmons, J., Namgaladze, A. A., Gustavsson, B., Wahlund, J.-E., and Eliasson, L., Yurik, R. Y.: Electron signatures and Alfvén waves, J. Geophys. Res., doi:10.1029/2001JA900096, 2002.

Cabrit, B., Opgenoorth, H., and Kofman, W.: Comparison between EISCAT UHF and VHF backscattering cross section, J. Geophys. Res., 101, 2369-2376, doi:10.1029/95JA02175, 1996.

Collis, P. N., Häggström, I., Kaila, K., and Rietveld, M. T.: EISCAT radar observations of enhanced incoherent scatter spectra; their relation to red aurora and field-aligned currents, Geophys. Res. Lett., 18, 1031-1034, 1991.

Cresswell, G.: Flaming auroras, J. Atmos. Terr. Phys., 31, 179-183, 1969.

Forme, F. R. E.: A new interpretation on the origin of enhanced ion acoustic fluctuations in the upper ionosphere, Geophys. Res. Lett., doi:10.1016/0021-9169(69)90092-0, 20, 2347-2350, 1993.

Forme, F. R. E.: Parametric decay of beam-driven Langmuir wave and enhanced ion-acoustic fluctuations in the ionosphere: a weak turbulence approach, Ann. Geophys., 17, 1172-1181, 1999,

\section{SRef-ID: 1432-0576/ag/1999-17-1172.}

Foster, J. C., del Pozo, C., Groves, K., and St. Maurice, J.-P.: Radar observations of the onset of current driven instabilities in the topside ionosphere, Geophys. Res. Lett., 15, 160-163, 1988.

Grydeland, T., La Hoz, C., Hagfors, T., Blixt, E. M., Saito, S., Strømme, A., and Brekke, A.: Interferometric observations of filamentary structures associated with plasma instability in the auroral ionosphere, Geophys. Res. Lett., 30, 1338, doi:10.1029/2002GL016362, 2003.

Grydeland, T., Blixt, E. M., Løvhaug, U. P., Hagfors, T., La Hoz, C., and Trondsen, T. S.: Interferometric radar observations of filamented structures due to plasma instabilities and their relation to dynamic auroral rays, Ann. Geophys., 22, 1115-1132, SRefID:1432-0576/ag/2004-22-1115, 2004.

Holt, J. M., Erickson, P. J., Gorczyca, A. M., and Grydeland, T.: MIDAS-W: a workstation-based incoherent scatter radar data acquisition system, Ann. Geophys., 18, 1231-1241, 2000,

SRef-ID: 1432-0576/ag/2000-18-1231

Ivchenko, N. and Marklund, G.: Observations of low frequency activity at $1000 \mathrm{~km}$ altitude, Ann. Geophys., 643-648, 2001.
Ivchenko, N. and Marklund, G.: Current singularities observed on Astrid-2, Adv. Space Res., 1779-1782, doi:10.1016/S02731177(02)00448-9, 2002.

Ivchenko, N., Rees, M., Lanchester, B., Galand, D. L. M., Throp, K., and Furniss, I.: Observations of $\mathrm{O}^{+}\left({ }^{4} \mathrm{P}_{-}{ }^{4} \mathrm{D}^{0}\right)$ lines in electron aurora over Svalbard, Ann. Geophys., 22, 2805-2817, 2004, SRef-ID: 1432-0576/ag/2004-22-2805.

Jussila, J., Holma, H., Kaila, K., Aikio, A., and Gallop, P.: High-resolution study of rayed auroral arcs, EISCAT Workshop, Menlo Park, 2003.

Lanchester, B., Rees, M., Robertson, S., Galand, D. L. M., Mendillo, M., Baumgardner, J., Furniss, I., and Aylward, A. D.: Proton and electron precipitation over Svalbard - first results from a new Imaging Spectrograph (HiTIES), in Proc. of Atmos. Studies by Optical Methods, 33-36, SGO Pubs 92, 2003.

Neubert, T. and Christiansen, F.: Small-scale field-aligned currents at the top-side ionosphere, Geophys. Res. Lett., 30, doi:10.1029/2003GL017808, 2003.

Omholt, A.: The Optical Aurora, Springer-Verlag, Berlin, 1971.

Rietveld, M. T., Collis, P. N., and St.-Maurice, J.-P.: Naturally enhanced ion acoustic waves in the auroral ionosphere observed with the EISCAT 933-MHz radar, J. Geophys. Res., 96, 1929119305, 1991.

Rietveld, M. T., Collis, P. N., van Eyken, A. P., and Løvhaug, U. P.: Coherent echoes during EISCAT UHF common programmes, J. Atmos. Terr. Phys., 58, 161-174, doi:10.1016/00219169(95)00027-5, 1996.

Scourfield, M. and Parsons, N.: Aurora pulsations and flaming some initial results fo a cinematographic study using an image intensifier, Planet. Space Sci., 17, 1141-1147, doi:10.1016/00320633(69)90006-3, 1969.

Sedgemore-Schulthess, F. and St.-Maurice, J.-P.: Naturally enhanced ion-acoustic spectra and their interpretation, Surveys in Geophysics, 22, 55-92,doi:10.1023/A:1010691026863, 2001.

Sedgemore-Schulthess, K. J. F., Lockwood, M., Trondsen, T. S., Lanchester, B. S., Rees, M. H., Lorentzen, D. A., and Moen, J.: Coherent EISCAT Svalbard Radar spectra from the dayside cusp/cleft and their implication for transient field-aligned currents, J. Geophys. Res., 104, 24 613-24 624, doi:10.1029/1999JA900276, 1999.

Stasiewicz, K., Bellan, P., Chaston, C., Kletzing, C., Lysak, R., Maggs, J., Pokhotelov, O., Seyler, C., Shukla, P., Stenflo, L., Streltsov, A., and Wahlund, J.: Small scale Alvénic structures in the aurora, Space Sci. Rev., 92, 423-533, doi:10.1023/A:1005207202143, 2000.

Wannberg, G., Wolf, I., Vanhainen, L.-G., Koskenniemi, K., Rttger, J., Postila, M., Markkanen, J., Jacobsen, R., Stenberg, A., Larsen, R., Eliassen, S., Heck, S., and Huuskonen, A.: The EISCAT Svalbard radar: a case study in modern incoherent scatter radar system design, Radio Science, 32, 2283-2307, doi:10.1029/97RS01803, 1997. 